\title{
Immediate Placement and Provisionalization of Maxillary Posterior Single Implants
}

\author{
Abu-Hussein Muhamad, Chlorokostas Georges, Watted Nezar, Abdulgani Mai, \\ Abdulgani Azzaldeen
}

\begin{abstract}
Functional immediate load is the most recent concept. This describes a load, within physiological limits, applied to implants before the osseo-integration process is completed. One of the treatment options offered to patients requiring replacement of one or more teeth is the use of prostheses supported by implants. Patients nowadays demand greater aesthetic and functional restorations; therefore, the clinician tries to reduce implant load time. All this leads to the implementation of several modifications to the conventional surgical and prosthetic protocol leading to a reduction in the load time of the implant. This is a case report of the immediate loading of posterior implant.
\end{abstract}

Keywords: Implants, primary stability, immediate loading, insertion torque.

\section{Introduction}

Endosseous dental implant therapy has become a widely accepted treatment modality for replacement of missing teeth. From the early beginnings of osseointegration, the field of implant dentistry has witnessed a number of paradigm shifts from the original implant placement protocols. [1]At that time, whenever a tooth was deemed hopeless and extraction the recommended course of therapy, the tooth would be removed, and a period of healing would be recommended prior to placement of an implant. Once healed, the clinician would then place the implant, submerging it under the gingival tissues, allowing integration of the implant prior to restoring. [2] A second stage surgery would then be performed to expose the implant, and prepare it for restoration. Due to a desire to increase the overall efficiency of the process, as well as an idea that outcomes may be improved, the concept of immediate placement and loading of implants began to emerge.[3] By placing an implant immediately after extraction, the overall treatment time would be shortened, theoretically increasing patient satisfaction and possible acceptance of treatment, as well as reducing overall costs incurred by the treating dentist. Soon, investigators began to discover that placement of a temporary restoration (immediate loading) might improve the overall esthetic outcome of implants placed in such a manner, as well as further shorten treatment time, by eliminating the need for an additional procedure to expose the implant (second stage surgery).[1,2,3]

Immediate implant loading can be briefly defined as the loading of a dental implant immediately or few hours after being placed. Misch and coworkers defined as immediate occlusal loading within two weeks of implant insertion. Wang et al defined Immediate implant loading as an implant-based surgical technique in which the "implant supported restoration is placed into occlusal loading within at least 48 hours after implant placement." On basis of the consensus obtained from International Congress of Oral Implantologists meeting at Naples (Italy) in May 2006. The terms ,,non-functional immediate loadinge and ,immediate restoration ${ }^{\text {ee }}$ are used when a prostheses is fixed to the implants within $72 \mathrm{hrs}$ without achieving full occlusal contact with the opposing dentition. [ 4,5$]$

In the early 1990s, the concept of "immediate loading" was introduced. Today, more than 15 years of clinical and histological investigations have demonstrated its virtues. [6,7] There is growing scientific evidence showing that osseointegration can be achieved even at implants placed in fresh extraction sockets . Another breakthrough in implant dentistry is the possibility of immediate loading of implants in freshly extracted tooth sockets. [8]The immediate occlusal-loading protocol is an implant-supported temporary or definitive restoration in occlusal contact within two weeks of the implant insertion.[6] Immediate loading of dental implant has gained popularity due to less tissue trauma, reduced overall treatment time, decreased patient's anxiety and discomfort, high patient acceptance and better function and aesthetics. 


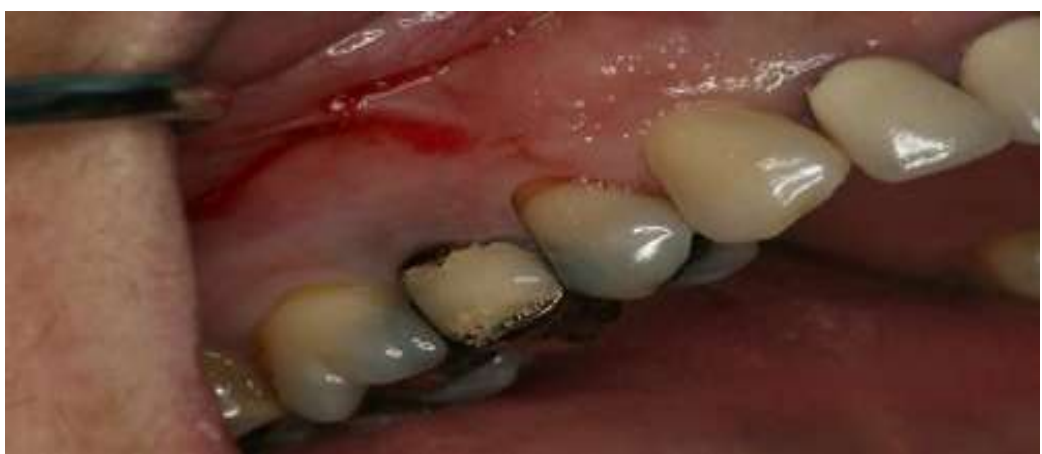

Fig.1; \#14 Presented With Vertical Fracture

Immediate loading has been used in the clinical routine of implant dentistry because of its high predictability. Using this technique, an osseointegrated implant can be implanted and loaded using a provisional structure so that function is restored immediately after the procedure. In addition to comfort and esthetics, there are psychological and functional advantages over the use of partial removable provisional prosthesis. [8,9] Provisional prostheses for immediate-loading implants may be manufactured using several techniques. The purpose of this prosthetic treatment step is not only the patient's social rehabilitation and well-being, but also the functional restoration of occlusion and esthetics as well as the preservation of an adequate gingival and bone structure. An alternative to provisional prostheses, particularly when treating the anterior maxilla, is the use of the crown of the fractured tooth. This technique may preserve original anatomy and provide good esthetic results, because the natural crown has the same color as the other teeth.[10,11] Several investigators have suggested the loading of implants at different intervals of time. Mish et al (2004) has classified the implant loading as - immediate occlusal loading, early occlusal loading, non- functional immediate restoration, nonfunctional early restoration and delayed occlusal loading. Immediate D direct loading: the provisional D definitive prosthetic construction is attached to the implant within 24 hours of the implant being placed. Whereas Early loading D Early functional loading: the provisional D definitive prosthetic construction is attached to the implant within days D weeks of the implant being placed.[5] Patients were ready for two stage surgical procedure and a long waiting period before they can begin to enjoy the benefits of the prosthesis for masticatory function, speech, appearance and self confidence. In the present days being edentulous for a long time is totally not acceptable for the patients.[12] The shorter the time required to replace the lost tooth greater is the acceptance to the patient. Conventional loading of an implant prosthesis would be for a period of 3-6 months where as immediate loading is done within 72 hours of implant placement. [13] This is a case report of the immediate loading of posterior implant.

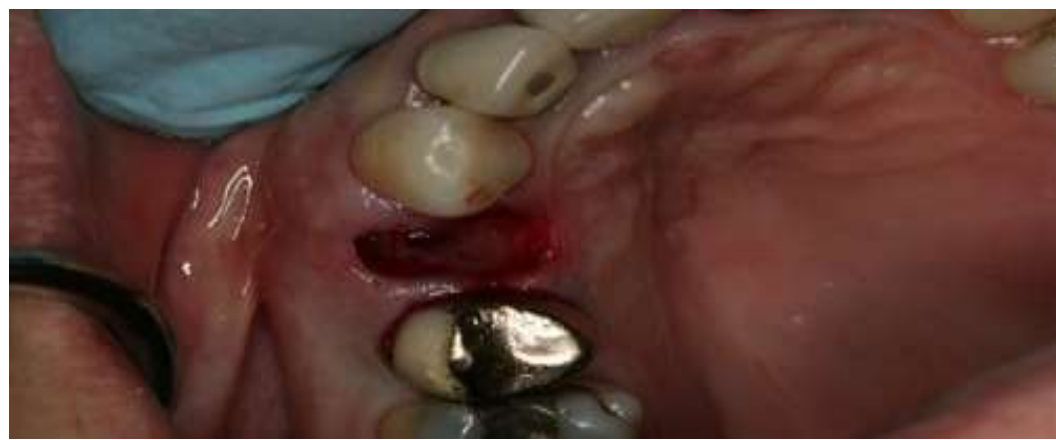

Fig.2; \#14 Or 1st Right Premolar For Extraction

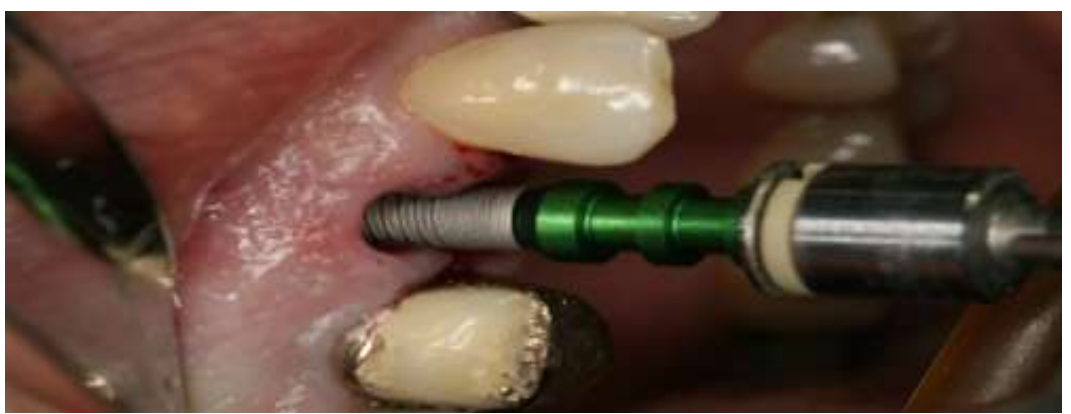

Fig. 3; Prosthetically Driven Placement 


\section{Case report}

A 48-year-old male patient presented with a history of trauma and crown fracture at the cervical area of tooth 14 and requested an immediate solution. Clinical and radiological evaluation revealed adequate alveolar bone, absence of periapical pathology but fracture line was below the crest of alveolar bone and was limited to the tooth. So, it was decided to extract and place endosseous implant immediately and place a provisional restoration to avail the benefits like preservation of bone and emergence profile. Fig. 1

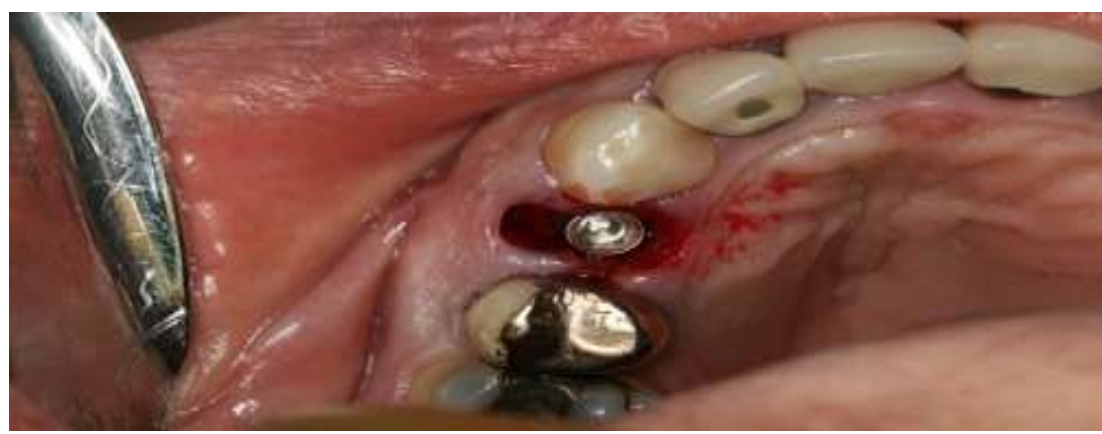

Fig. 4; Legacy Implant Placed

After administering appropriate antibiotic and analgesic, induction of local anaesthesia was carried out using xylocaime $2 \%$ with adrenaline $1 ; 200,000$. As preservation of alveolar bone is key to success of immediate implants, extraction of tooth has to be atraumatic, so using periotomes and small periosteal elevators the fragment was luxated without excessive enlargement of the socket, and using an innovative method where endodontic file was used to engage the canal wall and tooth fragment was slowly luxated and pulled out of the socket using the file . Fig. 2,3,4

Immediately after extraction, the socket was irrigated with saline solution and the walls were carefully examined to check their integrity on all surfaces. An osseointegrated implant was then placed respecting its correct three-dimensional positioning, a determinant factor to preserve gingival esthetics. Proper locking was achieve at over $40 \mathrm{~N} / \mathrm{cm} 2$, and the implant was immediately loaded.

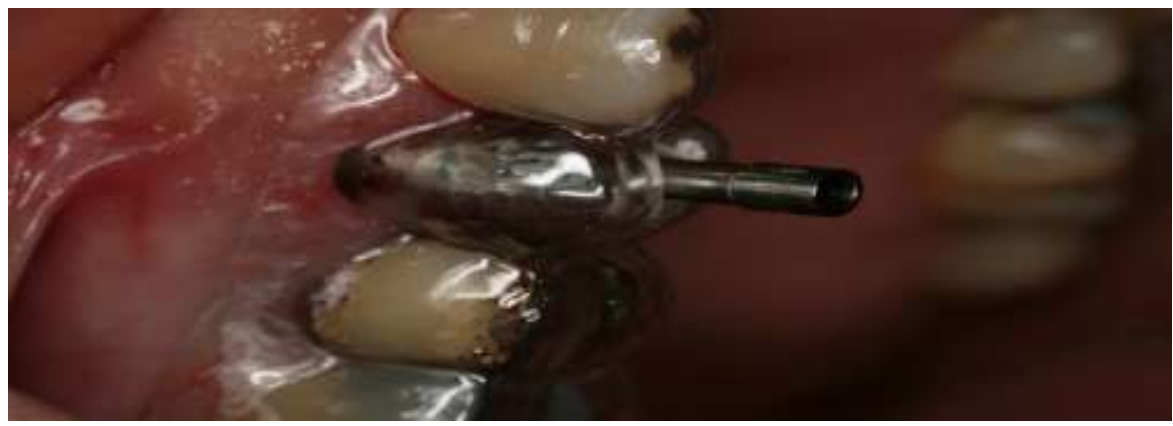

Fig. 5; Position Of Implant Respecting Prosthetic And Surgical Principles

To manufacture the implant-supported provisional restoration, the crown of tooth \#14was prepared into a facet , which was then rebased over a provisional using a

autopolymerizing acrylic resin . The quality of the polishing of autopolymerizing acrylic resin surfaces, when compared with that of acrylic resins, makes cleaning easier and, consequently, promotes gingival health in the region. Fig. 5,6

However, the cervical contour of the provisional is responsible for tissue stability at the gingiva-toothimplant interface.

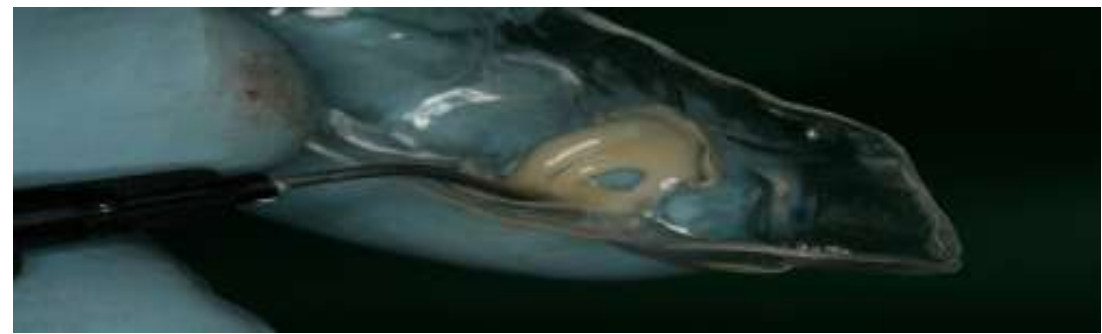

Fig. 6; The prefabricated provisional restoration was filled with autopolymerizing acrylic resin 


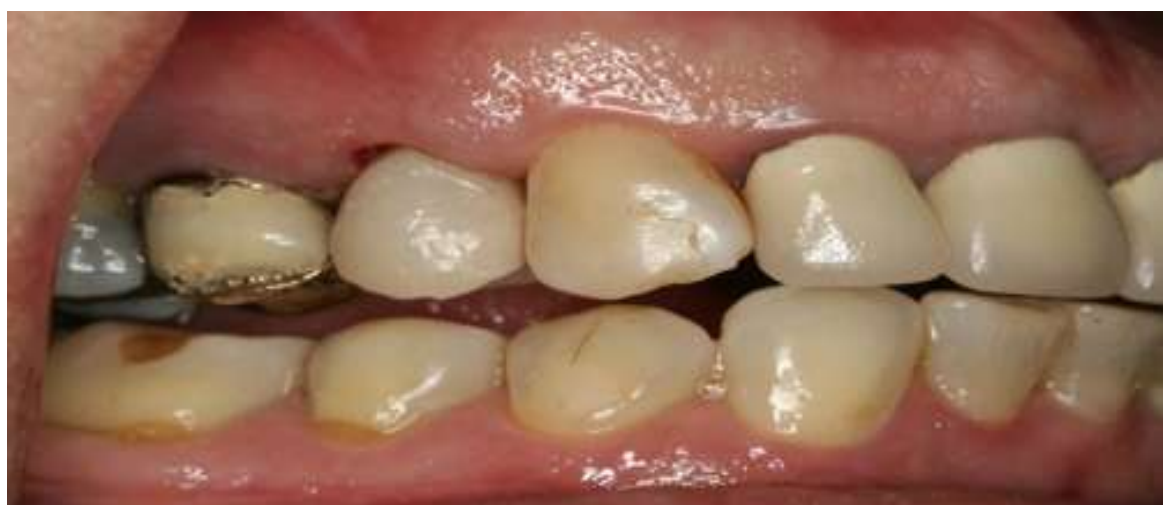

Fig. 7; Out of occlusion on lateral movements, immediate loaded provisional restoration

Occlusal adjustments were made in the provisional restoration to ensure that it was free of protrusive and lateroprotrusive contacts that might lead to trauma and complicate osseointegration during the primary periimplant bone remodeling phase and might, consequently, result in implant failure. Fig. 7

After 90 days, the definitive restoration was initiated. At that moment, the gingiva was healthy and the concave arch and gingival papillae were not negatively affected. Stability of peri-implant soft tissues and of emergence profile was favored by the use of the crown of the natural tooth and by its cervical anatomy.

\section{Discussion}

Fundamental requirement for immediate occlusal loading is its primary stability.[12,13] This primary stability is measured in units of insertion torque values or resonance frequency analysis. In yester years the stability of implants was achieved through a period of undisturbed healing. Newer concepts of immediate loading of the implant help to provide immediate prosthetic needs. Primary stability is now achieved via mechanical phenomenon of screw stability and splinting.

Shorter healing times may be appropriate in some circumstances.[14] Randomized controlled trials have shown that survival and success rates of immediately loaded implants in the edentulous mandible are comparable with conventionally loaded implants. 4 In most studies, good bone quality and primary implant stability has been mentioned as an important prognostic factor for the success of the procedure.5 Rough implant surfaces improve the survival rates of the immediately loaded implant.[15] Immediate loading protocol can be successful in judiciously selected cases where in high initial stability has been achieved in good bone volume and quantity. Hence, by the evolution of the concept of immediate loading longer duration of waiting period by the patient to receive a tooth can be avoided and the comfort, function and esthetics are achieved quite easily.[12,13,14,15,16]

Statistically, maxillary premolar are the teeth most often affected $(68 \%)$ in cases of dental trauma, particularly due to their natural projection in the anterior

maxilla, which makes them more susceptible to structural injury. Maxillary premolar are also affected by a high percentage of trauma and account for $24 \%$ of the cases. Several techniques are available for the restoration of a lost tooth, such as the use of fixed or removable prosthesis and, currently, tooth replacement using osseointegrated implants.[17,18]

The use of the immediate loading technique may be indicated for all cases in which the immediate application of loads to an implant is possible. However, to increase chances of success and to allow the use ofimmediate loading with a provisional, initial implant locking pressure should be high, about $40 \mathrm{Ncm}$.[18]

Therefore, the measurement of insertion torque at the time of implant placement should determine whether the prosthesis can be placed and the provisional crown fabricated immediately. Also, micro-movements generated by occlusion and mastication should be minimized to avoid intercuspation and eccentric contacts, and the provisional prosthesis should not be removed during the initial phases of peri-implant bone repair. Fig. 7 [17,18] The surgery protocol should include the evaluation of bone quality and quantity, as well as measures to ensure that the placement of the prosthesis and other components is adequate and to prevent parafunctional habits.[14] In the case described here, in addition to an initial locking pressure greater than $40 \mathrm{Ncm}$, careful provisional fabrication and adjustment ensured that the esthetic result was good and avoided centric and eccentric contacts.[18] In our case, the use of the palatalapproach preserved the buccal wall of the socket, which was filled only with the clot, and kept in place by the edge of the provisional prosthesis itself.[18]

One of the advantages of immediate loading is the restoration of function and esthetics immediately after surgery. Moreover, the adjacent gingival papillae are preserved and a second surgery is not necessary. [18]Also, the use of a removable provisional prosthesis for a long time while waiting for rehabilitation with a permanent prosthesis may be a problem, but not when the technique described 
here is used, because the provisional prosthesis is inserted and fixed immediately after surgery. In the clinical case described here, there was an important psychological gain as the use of a partial removable prosthesis was avoided .[14,18]

Clinically, the torque during implant placement is a good predictor of implant stability. Studies have reported that implants placed with an insertion torque greater than $30-35 \mathrm{Ncm}$ resulted in higher success rates for immediate loading. Additionally, to ensure adequate bone health andstability, proper implant placement technique includes copious irrigation both internally and externally to maintain temperatures

less than $47^{\circ} \mathrm{C}$ for prevention of necrosis of the surrounding bone[5].

The quality of bone often controls the prosthetic choices when immediate loading is considered. The need for bone grafting at the time of implant surgery may be necessary, depending on the anatomical variances of the patient' $s$ bony anatomy. Bone quality can be described in many ways. [20]The system proposed by Lekholm and Zarb places bone into four classifications based on the relative amounts of cortical and trabecular bone. In the first classifi cation almost the entirety

of bone is composed of compact cortical bone. In the second classification, compact trabecular bone is surrounded by a thick layer of cortical bone. [21] The third classifi cation is described as a thin layer of cortical bone encompassing high-density trabecular bone with favorable strength properties. Finally, in the fourth and least desirable bone type, a thin layer of compact bone surrounds loosely arranged trabecular bone. Higher failure rates have been reported in type IV bone for immediate loading of implants.[22]

The recommended occlusal scheme for immediately loaded implants is one of maximal interocclusal contacts without lateral contacts. [4] Patients with parafunctional habits or compromised occlusion should not receive immediate loading options. Studies by Balshi and Wolfinger demonstrated that approximately $75 \%$ of failures with immediate loading occurred in patients with parafunctional habits. Additional studies have supported these results and suggest that these patients, if not excluded from immediate loading, must be strongly cautioned of the high risk for failure.[23] Various studies have been done on these single-tooth restorations placed into immediate occlusion via provisionalization with success rates similar to those implants restored with light or no occlusal forces.[24] Other studies of these single-tooth restorations have shown lower success rates when placed into immediate functional occlusion. Clearly, more detailed studies are needed to assess the role of occlusion in these restorations. Fig. 7 [24,25] Studies of implants placed in type IV bone with varying degrees of success.[26,17] The soft tissue response was very favorable in these studies owing to the presence of a provisional crown throughout the healing phase, which allowed the sculpting of the interdental papilla and the attached gingiva.[26,27,28] In this treatment, an initial insertion torque of at least $45 \mathrm{Ncm}$ was reached. Although clinical studies on immediate single implant loading reported varying minimal insertion torques for immediate loading, the authors of this report adopted a threshold of $45 \mathrm{Ncm}$. [ 29]For this patient, a substantial maturation of the papillae occurred during the provisional phase. Care was taken to ensure that the provisional crown did not disturb this process, but served as a natural guide.

Regeneration of papillae with time has been reported in several studies,but the mechanism behind this phenomenon could not validly be explained. Some authors believe that this increase can be attributed to the remodeling potential of the soft tissues to establish a proper biological height after the surgical manipulation. [ 29,30 ] It is widely accepted, however, that the interproximal bone level next to the adjacent teeth is important for the future level of the interproximal papillae of the implant. [30] The immediate implant placement is an increasingly common strategy to preserve bone and reduce treatment times includes the placement of a dental implant into a recent extraction site. In addition, immediate loading is becoming more common as success rates for this procedure are now acceptable. [29,30] This can cut months off the treatment time and in some cases a prosthetic tooth can be attached to the implants at the same time as the surgery to place the dental implant.

\section{Conclusion}

Immediate implant placement into the fresh extraction sockets followed by functional immediate loading with provisional prosthesis on premolars for a patient having premolars protected occlusion is also a predictable treatment option if the patient's cooperation is assured.The immediate loading of single dental implant in maxillary posterior zone is a viable clinical concept and lead to favorable treatment outcomes. Practitioners have experienced less expense in the form of chair time and greater patient satisfaction.

\section{References}

[1]. Furze D, Byrne A, Donos N, Mardas N. Clinical and esthetic outcomes of single-tooth implants in the anterior maxilla. Quintessence Int. 2012;43(2):127-34

[2]. Chaushu G, Chaushu S, Tzohar A, Dayan D. Immediate loading of single-tooth implants: immediate versus non-immediate implantation. A clinical report. Int J Oral Maxillofac Implants. 2001;16(2):267-72.

[3]. Ericsson I, Nilson H, Lindh T, Nilner K, Randow K. Immediate functional loading of Brenemark single tooth implants. An 18 months' clinical pilot follow-up study. Clin Oral Implants Res. 2000;11(1):26-33.

[4]. Wang TM, Leu LJ, Wang J, Lin LD. Effects of prosthesis materials and prosthesis . splinting on peri-implant bone stress around implants in poor-quality bone: a numeric analysis. International Journal of Oral and Maxillofacial Implants. 2002;17(2): 231-7. 
[5]. Misch CE. Bone Density: A Key Determinant for Treatment Planning. In: Contemporary Implant Dentistry, (Misch CE) 3rd ed. Mosby Elsevier, St. Louis, Missouri;2008. pp. 130-146.

[6]. Abu-Hussein M., Abdulgani A., Watted N .Zahalka M.; Congenitally Missing Lateral Incisor with Orthodontics, Bone Grafting and Single-Tooth Implant: A Case Report. Journal of Dental and Medical Sciences2015, 14(4),124-130

[7]. Abdulgani A.,. Kontoes N., Chlorokostas G.,Abu-Hussein M .; Interdisciplinary Management Of Maxillary Lateral Incisors Agenesis With Mini Implant Prostheses: A Case Report; IOSR-JDMS 2015,14 (12) , 36-42

[8]. Abu-Hussein M., Abdulgani A., Watted N .Zahalka M.; Congenitally Missing Lateral Incisor with Orthodontics, Bone Grafting and Single-Tooth Implant: A Case Report. Journal of Dental and Medical Sciences2015, 14(4),124-130

[9]. Abu-Hussein M. , Abdulgani A., Bajali M., Chlorokostas G .; The Mandibular Two-Implant Overdenture.Journal of Dental and Allied Sciences , 2014, Vol 3,1; 58-62

[10]. Abdulgani Azzaldeen, Abusalih Ahmet ,Hakki Ismail ,Chlorokostas Georges, Abu-Hussein Muhamad; IMMEDIATE LOADING With Mini Dental Implants In The Fully Edentulous Mandible Int J Dent Health Sci 2015; 2(6):1071-1079

[11]. Abu-Hussein M. , Chlorokostas G ., Watted N ., Abdulgani Az ., Jabareen A.; Pre-Prosthetic Orthodontic Implant for Management of Congenitally Unerupted Lateral Incisors - A Case Report Journal of Dental and Medical Sciences 2016, 15, 2 , 99-104

[12]. Nezar Watted ,Mohamad Watad ,Abdulgani Azzaldeen, Abu-Hussein Muhamad; Immediate Anterior Dental Implant Placement: A Case Report .The Journal of Implant \& Advanced Clinical Dentistry2015Vol 7, 2, 11-19

[13]. Musa B, Azzaldeen A, Abu-Hussein M. . Immediate Implant Placement following tooth extraction A Case Report. International Journal of Dental Clinics.2014;6(3):19-20.

[14]. Muhamad A, Azzaldeen A, Aspasia SA, Nikos K. Implants into fresh extraction site: A literature review, case immediate placement report. J Dent Implant 2013;3:160-4.

[15]. M. Bajali, Azz. Abdulgani, M. Abu-Hussein, Anterior Maxillary Extraction, Immediate Implant Placement, and Provisionalization with Two Years Follow-up: A Case Report .The Journal of Implant \& Advanced Clinical Dentistry2014, Vol. 6, No. 6 ,5-11

[16]. Nezar Watted ,KareemTrabih ,Qawasmeh Nour , Abdulgani Azzaldeen2,Chlorokostas George ,Abu-Hussein Muhamad Extraction,Immediate Implant-A Case Report Int J Dent Health Sci 2014; 1(4): 679-685

[17]. Abdulgani Az . , Bajali M. and Abu-Hussein M .;Immediate Implant Placement With One Year Follow-Up: A CASE. International Journal of Basic and Applied Medical Sciences2013 Vol. 3 (3) , pp.189-197

[18]. Abu-Hussein M ., Chlorokostas G ., Abusalih A ., Ismail H ., Abdulgani Az . Immediate Implant Placement and Loading in Esthetic Zone Journal of Dental and Medical Sciences 2016, 15, Issue 1, PP 71-79

[19]. Abdulgani A, Bajali M. and Abu-Hussein M .;Immediate Implant Placement With One Year Follow-Up: A CASE. International Journal of Basic and Applied Medical Sciences2013 Vol. 3 (3) ,pp.189-197

[20]. Neugebauer J , Traini T , Thamus U, et al : Peri-implant bone organization under immediate loading state. Circularly polarized light analyses: a minipig study, J Periodontol 2006,77: $152-160$

[21]. Lekholm U, Zarb GA : Patient selection and preparation . In Branemark P-I , Zarb GA, Albrektsson T , editors: Tissue-integrated prosthesis: osseointegration in clinical dentistry, Chicago, 1985, Quintessence, pp199- 209.

[22]. Jaffi n RA, Berman CL : The excessive loss of Branemark fi xtures in type IV bone: a 5-year analysis, J Periodontol1991 . $62: 2$ -

[23]. Balshi TJ , Wolfi nger GJ : Immediate loading of Branemark implants in edentulous mandibles: a preliminary report, Implant Dent $1997.6: 83-88$

[24]. Glauser R, Lundgren AK, Gottlow J , et al : Immediate occlusal loading of Branemark TiUnite implants placed predominantly in soft bone: 1-year results of a prospective clinical study, Clin Implant Dent Relat Res2003.

[25]. $5: 47-56$

[26]. Calandriello R, Tomatis M , Rangert B : Immediate functional loading of Branemark system implants with enhanced initial stability: a prospective

[27]. to 2-year clinical and radiographic study, Clin Implant Dent Relat Res2003 . 5 ( Suppl ): 10 - 21

[28]. 26.Rocci A , Martignoni M , Gottlow $\mathbf{J}$ : Immediate loading in the maxilla using $\mathrm{fl}$ apless surgery, implants placed in predetermined positions, and prefabricated provisional restorations: a retrospective 3-year clinical study, Clin Implant Dent Relat Res2003, $5: 29$ $-36$

[29]. 27. Andersen E , Haanaes HR , Knutsen BM : Immediate loading of singletooth ITI implants in the anterior maxilla: a prospective 5-year pilot study, Clin Oral Implants Res $2002.13: 281-287$

[30]. Tarnow DP, Emitiaz S, Classi A : Immediate loading of threaded implants at stage 1 surgery in edentulous arches: ten consecutive case reports with to 5-year data, Int J Oral Maxillofac Implants1997 . $12: 319$ - 324

[31]. Azzaldeen A, Nikos K, Georgos C, Abu-Hussein M. ; Unilateral Maxillary Lateral Incisor Agenesis with Mini Implant Prostheses: A Case Report. Dent Implants Dentures2016, 1:106. doi:10.4172/did.1000106

[32]. Villa R, Rangert B. Immediate and early function of implants placed in extraction sockets of maxillary infected teeth: a pilot study. J Prosthet Dent 2007:97:S96-S108. 